\section{Predictors of 30-day post-discharge unplanned readmission in a subacute geriatric ward in Singapore}

\author{
Christine Yuanxin Chen ${ }^{1}$, MBBS, MRCP; Thulasi Chandran ${ }^{2}$, MBBS, FCFP; Pei \\ Ting Tan ${ }^{3}$, MHS; Vivian Cantiller Barrera ${ }^{1}$, MD, GDGRM; Rachelle Tumbokon \\ Tan-Pantanao ${ }^{1}$, MD, GDGRM; Quicho Tanya Joy Zapata ${ }^{1}$, MD, GDGRM; Thant \\ Zin Tun ${ }^{1}$, MBBS, GDGRM; Siti Humaira Binte Mohd Kamil'; Josceline Qiao Lin \\ Gan'; Kiat Sern Goh ${ }^{1}$, MBBS, MRCP
}

\begin{abstract}
Background. Unplanned readmission to hospital is common among older adults and contributes to considerable healthcare costs and hospital-associated complications. We aimed to identify predictors of 30-day post-discharge unplanned readmission among older adults in our subacute geriatric ward, and to determine the prevalence of geriatric syndromes and develop a new predictive model for readmission of subacute geriatric patients.
\end{abstract}

Methods: Consecutive patients admitted to our subacute geriatric ward between June 2018 and June 2019 were invited to participate. Data collected included patient age, sex, weight, height, race, type of housing, destination upon discharge, functional and frailty status, presence of conduits (urinary catheters and nasogastric tubes), polypharmacy, high-risk medications, healthcare utilisation 6 months prior, laboratory test results, length of hospital stay, Charlson Comorbidity Index, and LACE index. Patients were assessed using the Mini Nutritional Assessment -Short Form, Geriatric Depression Scale, Mini-Mental State Examination, Clinical Frailty Scale, FRAIL scale, modified Barthel Index, hand grip strength, and gait speed. Patients with or without 30-day post-discharge unplanned readmission were compared. Multivariate logistic regression was used to identify independent predictors.

Results: Of 284 patients followed up at 30 days post-discharge, 63 (22.2\%) had unplanned hospital readmission within 30 days of discharge, with associated factors being history of myocardial infarction, moderate or severe liver or renal disease, low albumin level, history of emergency department visits, hospitalisation in the preceding 6 months, and discharge to a destination other than home. The prevalence of geriatric syndromes of falls, frailty, and immobility was $62.3 \%, 64.7 \%$, and $86.6 \%$, respectively. Independent predictors of 30-day post-discharge unplanned readmission were history of hospitalisation in the preceding 6 months (odds ratio $=2.62, \mathrm{p}=0.045$ ) and discharge destination other than home (odds ratio=3.10, $\mathrm{p}=0.006$ ). The area under the receiver operating characteristics curve for the predictive models was between 0.6 and 0.7 , and Brier score was around 0.16 . The discrimination ability of the models was weak.

Conclusion: History of hospitalisation in the preceding 6 months and not being discharged to home were independent predictors for 30-day post-discharge unplanned readmission.

Key words: Aged; Patient readmission; Risk factors; Subacute care

\section{ORIGINAL ARTICLE}

Department of Geriatric Medicine, Changi General Hospital, Singapore

2 Medical Service, Saint Andrew's Community Hospital, Singapore

Clinical Trials Research Unit, Changi General Hospital, Singapore

4 Yong Loo Lin School of Medicine, National University of Singapore, Singapore
Correspondence to: Christine Yuanxin Chen, Department of Geriatric Medicine, Changi General Hospital, Singapore. Email: christine.chen.y.x@singhealth.com.sg 


\section{INTRODUCTION}

Unplanned readmission to hospital is common among older adults and contributes to escalating healthcare expenditure, hospital bed occupancy, caregiver burden, and poorer quality of life. Predictors of hospital readmission have been used to identify high-risk patients for interventions to minimise adverse health outcome. ${ }^{1-8}$

In 2018 , individuals aged $\geq 65$ years were involved in $29.8 \%$ of all admissions to acute hospitals and $80.8 \%$ of all admissions to community hospitals in Singapore. ${ }^{9}$ There is a need to cater for a subset of older patients who have been hospitalised and no longer need acute care, yet would not be suitable for sole rehabilitation. Our subacute ward was set up in a community hospital to accommodate this subset of patients who needed inpatient medical care during the treatment and recovery phase of an acute illness, injury, or exacerbation of a known chronic disease. ${ }^{10}$ Patients generally stay for 2 to 4 weeks, and emphasis is placed on achieving rehabilitation goals and early care discharge planning to ensure a smooth discharge to the community. Our subacute ward has 30 beds and receives an average of 33 to 35 new admissions each month. Admissions come from the acute geriatric wards, non-geriatric wards, and the emergency department short-stay unit.

We aimed to identify predictors of 30-day post-discharge unplanned readmission among older adults in our subacute geriatric ward, and to determine the prevalence of geriatric syndromes and develop a new predictive model for readmission of subacute geriatric patients.

\section{METHODS}

This prospective study was carried out from June 2018 to June 2019. Consecutive patients admitted to our subacute geriatric ward and consented to participate were recruited. Patients who died during the subacute inpatient stay were excluded. Informed consent was obtained from each patient. If the patient was uncommunicative or unable to give consent, proxy consent was obtained from their next-of-kin. Ethics approval was obtained from the
Singhealth Centralised Institutional Review Board, Singapore (CIRB Ref: 2017/3000).

Data collected from electronic health records included patient age, sex, weight, height, race, type of housing, destination upon discharge, functional and frailty status, the number of medications on discharge, use of high-risk medications (anticoagulants, dual antiplatelet therapy), use of other medications (anxiolytics, insulin, antipsychotics, antidepressants, and diuretics), laboratory test results (serum sodium, haemoglobin, and albumin levels and estimated glomerular filtration rate), the requirement of feeding tubes and urinary catheters during admission, continence status on discharge, length of stay in the acute ward, length of stay during subacute admission, any delay in discharge and reasons, and healthcare utilisation 6 months prior (visit to emergency department and any hospital admission).

Patients were assessed using various instruments. The Mini Nutritional Assessment -Short Form ${ }^{11}$ comprises six questions on food intake, weight loss, mobility, psychological stress or acute disease, the presence of dementia or depression, and body mass index; scores of $\geq 12$ indicate acceptable nutritional status. The Geriatric Depression Scale comprises 15 questions to screen for the presence of depression; scores of $\geq 5$ indicate the possible presence of depression. The Mini-Mental State Examination ${ }^{12}$ was used to screen for cognitive impairment; the cut-off of normal cognitive function is usually set as 24, although more advanced age and fewer years of formal education may allow for a lower cut-off. The Clinical Frailty Scale ${ }^{13}$ was used by a clinician to interview patients and families to determine their baseline score prior to admission; scores range from 1 (very fit) to 9 (terminally ill) and were categorised as non-frail (1-3), pre-frail (4), and frail (>4) for data analysis. The fatigue, resistance, ambulation, illnesses and loss of weight (FRAIL) scale ${ }^{14}$ was used as comparison. The modified Barthel Index ${ }^{15}$ was used to assess ability to perform activities of daily living (eg, personal care, mobility, transfers, bathing, and feeding) on admission and at discharge. Hand grip strength ${ }^{16}$ was measured by an occupational therapist using the Jamar digital dynamometer, with the arm at right angles and the elbow by the side of the body. Two attempts from each hand were 
recorded, with at least $15 \mathrm{~s}$ for recovery allowed between each attempt. Gait speed ${ }^{17}$ was measured by a physiotherapist for patients who required most standby assistance for ambulation with or without aids. It is the time taken to ambulate 4 metres on a level surface. A taped line was placed 1 metre before and after the 4 metres walkway for acceleration and deceleration. The Charlson Comorbidity Index ${ }^{18}$ and the LACE index ${ }^{19}$ (length of stay, acuity of admission, Charlson Comorbidity Index, and number of emergency department visits in the preceding 6 months) were generated.

Patients with or without 30-day post-discharge unplanned readmission were compared using Chi squared test or Fisher exact test for categorical variables and independent $t$-test or Mann-Whitney $U$ test for numerical variables. Significant variables in univariate analysis were included in the multivariate logistic regression model to identify the independent predictors. Variables with good model fit $(p>0.05$ in Hosmer-Lemeshow goodness-of-fit test) were further tested for their ability to discriminate the case from non-case using receiver operating characteristics (ROC) curve analysis. ROC analysis was performed to determine the optimal cut-off point of the Youden index in differentiating patients with 30-day post-discharge unplanned readmission. Overall performance of four prediction models was evaluated using the Brier score. Models with different combinations of independent predictors were assessed using the Hosmer-Lemeshow goodnessof-fit test. All models were compared using ROC curve analysis in terms of predicting 30-day postdischarge unplanned readmission. Statistical analysis was performed using SPSS (version 20.0, IBM, Armonk [NY], USA). A P value of $<0.05$ was considered statistically significant.

\section{RESULTS}

Of 400 patients screened, 301 were eligible and consented to participate. Of whom, 284 (94.4\%) were followed up at 30 days post-discharge. Of the 284 patients, 252 (89\%) were admitted from an acute hospital ward with a median length of stay of 11 days and $30(10.6 \%)$ were from the short stay unit of the emergency department with a median length of stay of 19 days. Of the 284 patients, 63 (22.2\%) had unplanned hospital readmission within 30 days of discharge. Those with or without 30-day postdischarge unplanned readmission were comparable in terms of baseline characteristics (TABLE $\mathbf{1}$ ).

In univariate analysis, 30-day post-discharge unplanned readmission was associated with a history of myocardial infarction (odds ratio $[\mathrm{OR}]=1.91$, $\mathrm{p}=0.035)$, the presence of moderate or severe liver or renal disease $(\mathrm{OR}=3.86, \mathrm{p}=0.010)$, the Charlson Comorbidity Index $(\mathrm{OR}=1.20, \mathrm{p}=0.001)$, low serum albumin level $(\mathrm{OR}=2.02, \mathrm{p}=0.018)$, the LACE index $(\mathrm{OR}=1.18, \mathrm{p}=0.004)$, emergency department visits in the prior 6 months $(\mathrm{OR}=1.77, \mathrm{p}=0.049)$, hospitalisation in the prior 6 months $(\mathrm{OR}=2.42$, $\mathrm{p}=0.002)$, and discharge destination other than home (OR=2.68, $\mathrm{p}=0.008)$ [TABLE 2].

In multivariate logistic regression, independent predictors of 30-day post-discharge unplanned readmission were hospitalisation in the prior 6 months $(\mathrm{OR}=2.62, \mathrm{p}=0.045)$ and discharge destination other than home $(\mathrm{OR}=3.10, \mathrm{p}=0.006)$ [TABLE 2].

Hosmer-Lemeshow goodness-of-fit test showed that a model with the LACE index only was a poor fit model $(\mathrm{p}=0.003)$. Using the Youden Index to determine the optimal cut-off point, sensitivity, specificity, and negative and positive predictive values of the remaining four models are shown in TABLE 3. The area under the ROC curve of the four models ranged from 0.6 to 0.7 , and the Brier score was around 0.16 , which indicated that none of the models had excellent discrimination ability (FIGURE).

The prevalence of geriatric syndromes was high: $17.7 \%$ for delirium, $30 \%$ for dementia $(21.2 \%$ of whom had moderate to severe dementia), $16.3 \%$ for depression, $62.2 \%$ for a history of falls, $44.5 \%$ for osteoporosis, $41.3 \%$ for urinary incontinence, and $86.1 \%$ for functional decline.

Based on the FRAIL scale, $7.1 \%$ of patients were robust (score of 0 ), $33.6 \%$ were pre-frail (score of $1-2$ ), $40.6 \%$ were frail (score of $\geq 3$ ), and $18.7 \%$ did not have FRAIL score. Using the Clinical Frailty Scale, $61.1 \%$ were frail (score of $\geq 5$ ) and $37.1 \%$ were non-frail. 
TABLE 1

Patient demographic and clinical characteristics

\begin{tabular}{|c|c|c|c|c|}
\hline \multirow[t]{2}{*}{ Parameter } & \multirow[t]{2}{*}{ All $(n=284)^{*}$} & \multicolumn{2}{|c|}{ 30-day post-discharge unplanned readmission } & \multirow[t]{2}{*}{$P$ value } \\
\hline & & No $(n=221)^{\star}$ & Yes $(n=63)^{\star}$ & \\
\hline \multicolumn{5}{|l|}{ Demographic } \\
\hline Age, y & $83.4 \pm 7.1(66-105)$ & $83.7 \pm 7.1(66-105)$ & $82.7 \pm 7.2(69-100)$ & 0.334 \\
\hline Ethnicity & & & & 0.236 \\
\hline Chinese & 207 (72.9) & $163(73.8)$ & $44(69.8)$ & \\
\hline Malay & $59(20.8)$ & $42(19.0)$ & $17(27.0)$ & \\
\hline Others & $18(6.3)$ & $16(7.2)$ & $2(3.2)$ & \\
\hline Sex & & & & 0.069 \\
\hline Male & $116(40.9)$ & $84(38.0)$ & $32(50.8)$ & \\
\hline Female & $168(59.1)$ & $137(72.0)$ & $31(49.2)$ & \\
\hline Housing type & & & & 0.262 \\
\hline Private & 30 (10.6) & $23(10.5)$ & $7(11.1)$ & \\
\hline Public (Housing Development Board flats) & $243(86.2)$ & $187(85.4)$ & $56(88.9)$ & \\
\hline Others & $9(3.2)$ & $9(4.1)$ & $0(0.0)$ & \\
\hline Own a house & & & & 0.412 \\
\hline No & $20(7.3)$ & $14(6.6)$ & $6(9.7)$ & \\
\hline Yes & $253(92.7)$ & $197(93.4)$ & $56(90.3)$ & \\
\hline Weight, kg & $52.4 \pm 13.4(23.9-96.7)$ & $52.5 \pm 13.5(27.2-96.7)$ & $52.4 \pm 13.5(23.9-96.2)$ & 0.957 \\
\hline Height, cm & $152.9 \pm 11.3(98-181)$ & $152.9 \pm 10.9(120-181)$ & $153 \pm 12.7(98-176)$ & 0.978 \\
\hline Body mass index, $\mathrm{kg} / \mathrm{m}^{2}$ & $22.8 \pm 6(12.98-47.27)$ & $22.8 \pm 5.7(13-43.9)$ & $23.3 \pm 7(13.7-47.3)$ & 0.553 \\
\hline Nasogastric tube on admission & & & & 1.000 \\
\hline No & 278 (97.9) & $216(97.7)$ & $62(98.4)$ & \\
\hline Yes & $6(2.2)$ & $5(2.3)$ & $1(1.6)$ & \\
\hline Indwelling urinary catheter on admission & & & & 0.337 \\
\hline No & $268(94.7)$ & $210(95.5)$ & $58(92.1)$ & \\
\hline Yes & $15(5.3)$ & $10(4.5)$ & $5(7.9)$ & \\
\hline \multicolumn{5}{|l|}{ Comorbidity } \\
\hline Charlson Comorbidity Index & $3.3 \pm 2.6(0-13)$ & $3 \pm 2.4(0-10)$ & $4.4 \pm 3.1(0-13)$ & $<0.001$ \\
\hline Previous myocardial infarct & & & & 0.034 \\
\hline No & 209 (73.9) & $169(76.8)$ & $40(63.5)$ & \\
\hline Yes & $74(26.1)$ & $51(23.2)$ & $23(36.5)$ & \\
\hline Cerebrovascular disease & & & & 0.376 \\
\hline No & $162(57.3)$ & $129(58.6)$ & $33(52.4)$ & \\
\hline Yes & $121(42.7)$ & $91(41.4)$ & $30(47.6)$ & \\
\hline Peripheral vascular disease & & & & 0.274 \\
\hline No & $257(90.9)$ & $202(91.8)$ & $55(87.3)$ & \\
\hline Yes & $26(9.1)$ & $18(8.2)$ & $8(12.7)$ & \\
\hline Diabetes without complication & & & & 0.338 \\
\hline No & 248 (87.6) & 195 (88.6) & $53(84.1)$ & \\
\hline Yes & $35(12.4)$ & $25(11.4)$ & $10(15.9)$ & \\
\hline Congestive heart failure & & & & 0.048 \\
\hline No & 242 (85.5) & $193(87.7)$ & $49(77.8)$ & \\
\hline Yes & $41(14.5)$ & $27(12.3)$ & $14(22.2)$ & \\
\hline Diabetes with end organ damage & & & & 0.146 \\
\hline No & $217(76.7)$ & $173(78.6)$ & $44(69.8)$ & \\
\hline Yes & $66(23.4)$ & $47(21.4)$ & $19(30.2)$ & \\
\hline Chronic obstructive pulmonary disease & & & & 0.761 \\
\hline No & 267 (94.3) & $208(94.5)$ & $59(96.7)$ & \\
\hline Yes & $16(5.7)$ & $12(5.5)$ & $4(26.3)$ & \\
\hline
\end{tabular}

* Data are presented as mean \pm standard deviation (range), median (interquartile range), or No. (\%) of cases 
TABLE 1 (cont'd)

\begin{tabular}{|c|c|c|c|c|}
\hline \multirow[t]{2}{*}{ Parameter } & \multirow[t]{2}{*}{ All $(n=284)^{\star}$} & \multicolumn{2}{|c|}{ 30-day post-discharge unplanned readmission } & \multirow[t]{2}{*}{$P$ value } \\
\hline & & No $(n=221)^{\star}$ & Yes $(n=63)^{\star}$ & \\
\hline Mild liver or renal disease & & & & 0.566 \\
\hline No & $223(78.8)$ & $175(79.5)$ & 48 (76.2) & \\
\hline Yes & $60(21.2)$ & $45(20.5)$ & $15(23.8)$ & \\
\hline Any tumour & & & & 0.562 \\
\hline No & $265(93.6)$ & $207(94.1)$ & $58(92.1)$ & \\
\hline Yes & $18(6.4)$ & $13(5.9)$ & $5(7.9)$ & \\
\hline Dementia & & & & 0.946 \\
\hline No & $220(77.4)$ & $171(77.4)$ & $49(77.8)$ & \\
\hline Yes & $64(22.6)$ & $50(22.6)$ & $14(22.2)$ & \\
\hline Connective tissue disease & & & & 0.049 \\
\hline No & $281(99.2)$ & $220(100.0)$ & $61(96.8)$ & \\
\hline Yes & $2(0.8)$ & $0(0.0)$ & $2(3.2)$ & \\
\hline AIDS & & & & - \\
\hline No & $283(100.0)$ & $220(100.0)$ & $63(100.0)$ & \\
\hline Yes & $0(0.0)$ & $0(0.0)$ & $0(0.0)$ & \\
\hline Moderate or severe liver or renal disease & & & & 0.011 \\
\hline No & $267(94.3)$ & $212(96.4)$ & $55(87.3)$ & \\
\hline Yes & $16(5.7)$ & $8(3.6)$ & $8(12.7)$ & \\
\hline Metastatic solid tumour & & & & 0.400 \\
\hline No & 278 (99.2) & $216(99.5)$ & $62(98.4)$ & \\
\hline Yes & $2(0.8)$ & $1(0.5)$ & $1(1.6)$ & \\
\hline \multicolumn{5}{|l|}{ Medication } \\
\hline Anti-coagulant drug & & & & 0.975 \\
\hline No & 225 (79.5) & $175(79.5)$ & $50(79.4)$ & \\
\hline Yes & 58 (20.5) & 45 (20.5) & $13(20.6)$ & \\
\hline Anti-depression drug & & & & 0.773 \\
\hline No & $215(75.9)$ & $168(76.4)$ & $47(74.6)$ & \\
\hline Yes & $68(24.1)$ & $52(23.6)$ & $16(25.4)$ & \\
\hline Insulin & & & & 0.091 \\
\hline No & $269(95.0)$ & $212(96.4)$ & $57(90.5)$ & \\
\hline Yes & $14(5.0)$ & 8 (3.6) & $6(9.5)$ & \\
\hline Diuretics & & & & 0.850 \\
\hline No & $249(87.9)$ & $194(88.2)$ & $55(87.3)$ & \\
\hline Yes & $34(12.1)$ & $26(11.8)$ & $8(12.7)$ & \\
\hline Had more than 5 medications & & & & 0.521 \\
\hline No & $14(5.0)$ & $10(4.5)$ & $4(6.3)$ & \\
\hline Yes & $269(95.0)$ & $210(95.5)$ & $59(93.7)$ & \\
\hline \multicolumn{5}{|l|}{ Blood test } \\
\hline Serum sodium, mmol/l & $138.8 \pm 3.9(126-149)$ & $139 \pm 3.7(126-147)$ & $138.3 \pm 4.8(127-149)$ & 0.197 \\
\hline Serum creatinine, mg/dl & $79(61-106)$ & $78(60.3-106.8)$ & $87(63-106)$ & 0.182 \\
\hline Serum haemoglobin, g/dl & $11(9.8-12.2)$ & $11.1(10-12.2)$ & $10.2(9.1-11.8)$ & 0.002 \\
\hline Haemoglobin status & & & & 0.075 \\
\hline Normal & 79 (27.9) & $67(30.5)$ & $12(19.0)$ & \\
\hline Low & $204(72.1)$ & $153(69.5)$ & $51(81.0)$ & \\
\hline Serum albumin, g/l & $36.2 \pm 6.1(9.2-60)$ & $36.7 \pm 6.2(9.2-60)$ & $34.3 \pm 5.9(17-46)$ & 0.006 \\
\hline Albumin status & & & & 0.016 \\
\hline Normal ( $\geq 37$ ) & $140(49.8)$ & $117(53.7)$ & $23(36.5)$ & \\
\hline $\operatorname{Low}(<37)$ & $141(50.2)$ & $101(46.3)$ & $40(63.5)$ & \\
\hline Estimated glomerular filtration rate, $\mathrm{ml} / \mathrm{min}$ & $63.0(45-81)$ & $65.5(48.3-81.8)$ & $59(39-81)$ & 0.145 \\
\hline
\end{tabular}


TABLE 1 (cont'd)

\begin{tabular}{|c|c|c|c|c|}
\hline \multirow[t]{2}{*}{ Parameter } & \multirow[t]{2}{*}{ All $(n=284)^{\star}$} & \multicolumn{2}{|c|}{ 30-day post-discharge unplanned readmission } & \multirow[t]{2}{*}{$P$ value } \\
\hline & & No $(n=221)^{\star}$ & Yes $(n=63)^{*}$ & \\
\hline \multicolumn{5}{|l|}{ Scale } \\
\hline LACE index & $12.9 \pm 2.7(5-21)$ & $12.7 \pm 2.7(5-21)$ & $13.8 \pm 2.7(9-19)$ & 0.003 \\
\hline LACE cut-off at 9 & & & & 0.145 \\
\hline No risk $(\leq 9)$ & $27(9.5)$ & $24(10.9)$ & $3(4.8)$ & \\
\hline High risk (>9) & $257(90.5)$ & $197(89.1)$ & $60(95.2)$ & \\
\hline FRAIL Scale & & & & 0.439 \\
\hline Robust (0/5) & $20(8.6)$ & $14(7.7)$ & $6(12.5)$ & \\
\hline Pre-frail $(1-2 / 5)$ & $96(41.6)$ & $79(43.1)$ & $17(35.4)$ & \\
\hline Frail $(3-5 / 5)$ & $115(49.8)$ & $90(49.2)$ & $25(52.1)$ & \\
\hline Clinical Frailty Scale & & & & 0.455 \\
\hline Non-frail $(<4)$ & $52(18.6)$ & $43(19.7)$ & $9(14.8)$ & \\
\hline Pre-frail (=4) & $54(19.4)$ & $44(20.2)$ & $10(16.4)$ & \\
\hline Frail $(>4)$ & $173(62.0)$ & $131(60.1)$ & $42(68.8)$ & \\
\hline Mini Nutritional Assessment -Short Form & & & & 0.687 \\
\hline Normal (12-14) & $14(5.9)$ & $12(6.5)$ & $2(3.9)$ & \\
\hline At risk for malnutrition (8-11) & $107(45.3)$ & $85(45.9)$ & $22(43.2)$ & \\
\hline Malnourished (0-7) & $115(48.8)$ & $88(47.6)$ & $27(52.9)$ & \\
\hline Geriatric Depression Scale & & & & 0.983 \\
\hline Score $\geq 5$ & $103(47.7)$ & $81(47.4)$ & $22(47.8)$ & \\
\hline Score $<5$ & $113(52.3)$ & $89(52.6)$ & $24(52.2)$ & \\
\hline Mini-Mental State Examination & & & & 0.859 \\
\hline Score $\geq 23$ & $68(28.5)$ & $55(28.2)$ & $13(29.5)$ & \\
\hline Score $<23$ & $171(71.5)$ & $140(71.8)$ & $31(70.5)$ & \\
\hline \multicolumn{5}{|l|}{ Geriatric syndrome } \\
\hline Cognitive impairment & & & & 0.338 \\
\hline No & $203(71.5)$ & $161(72.8)$ & $42(66.7)$ & \\
\hline Yes & $81(28.5)$ & $60(27.2)$ & $21(33.3)$ & \\
\hline Dementia & 284 & 221 & 63 & 0.988 \\
\hline No & $198(69.7)$ & $154(69.7)$ & $44(69.8)$ & \\
\hline Yes & $86(30.3)$ & 67 (30.3) & $19(30.2)$ & \\
\hline $\begin{array}{l}\text { Behavioural and pyschological symptoms of } \\
\text { dementia }\end{array}$ & & & & 0.233 \\
\hline No & $260(91.5)$ & $200(90.5)$ & $60(95.2)$ & \\
\hline Yes & $24(8.5)$ & $21(9.5)$ & $3(4.8)$ & \\
\hline Delirium & & & & 0.389 \\
\hline No & $233(82.0)$ & $179(81.0)$ & $54(85.7)$ & \\
\hline Yes & $51(18.0)$ & $42(19.0)$ & $9(14.3)$ & \\
\hline Depression & & & & 0.214 \\
\hline No & $238(83.8)$ & $182(82.4)$ & $56(88.9)$ & \\
\hline Yes & 46 (16.2) & $39(17.6)$ & $7(11.1)$ & \\
\hline Falls & & & & 0.363 \\
\hline No & $104(37.0)$ & $78(35.6)$ & $26(41.9)$ & \\
\hline Yes & $177(63.0)$ & $141(64.4)$ & $36(58.1)$ & \\
\hline Osteoporosis & & & & 0.263 \\
\hline No & $116(47.9)$ & 87 (46.0) & $29(54.7)$ & \\
\hline Yes & $126(52.1)$ & $102(54.0)$ & $24(45.3)$ & \\
\hline Frailty & & & & 0.353 \\
\hline No & 100 (35.3) & 75 (33.9) & 25 (40.3) & \\
\hline Yes & $183(64.7)$ & $146(66.1)$ & $37(59.7)$ & \\
\hline Incontinence & & & & 0.635 \\
\hline No & 164 (58.2) & $129(58.9)$ & 35 (55.6) & \\
\hline Yes & $118(41.8)$ & $90(41.1)$ & $28(44.4)$ & \\
\hline
\end{tabular}


TABLE 1 (cont'd)

\begin{tabular}{|c|c|c|c|c|}
\hline \multirow[t]{2}{*}{ Parameter } & \multirow[t]{2}{*}{ All $(n=284)^{*}$} & \multicolumn{2}{|c|}{ 30-day post-discharge unplanned readmission } & \multirow[t]{2}{*}{$P$ value } \\
\hline & & No $(n=221)^{\star}$ & Yes $(n=63)^{*}$ & \\
\hline Immobility & & & & 0.821 \\
\hline No & $38(13.4)$ & $29(13.2)$ & $9(14.3)$ & \\
\hline Yes & $245(86.6)$ & $191(86.8)$ & $54(85.7)$ & \\
\hline \multicolumn{5}{|l|}{ Pre-admission condition } \\
\hline $\begin{array}{l}\text { History of emergency department visits in prior } \\
6 \text { months }\end{array}$ & & & & 0.048 \\
\hline No & $159(56.2)$ & $131(59.3)$ & $28(45.2)$ & \\
\hline Yes & $124(43.8)$ & $90(40.7)$ & $34(54.8)$ & \\
\hline History of hospitalisation in prior 6 months & & & & 0.002 \\
\hline No & $171(60.4)$ & $144(65.2)$ & $27(43.5)$ & \\
\hline Yes & $112(39.6)$ & $77(34.8)$ & $35(56.5)$ & \\
\hline \multicolumn{5}{|l|}{ Admission and discharge } \\
\hline Subacute admission from & & & & 0.463 \\
\hline Acute ward & $253(89.4)$ & $196(86.7)$ & $57(91.9)$ & \\
\hline Emergency department short stay unit & $30(10.6)$ & $25(11.3)$ & $5(8.1)$ & \\
\hline Length of acute stay & $11(7-18)$ & $11(7-17)$ & $13(7-21)$ & 0.136 \\
\hline Length of subacute stay & $19(14-27)$ & $19(14-25.8)$ & $21.5(15-30)$ & 0.216 \\
\hline Extension of stay beyond 28 days & & & & 0.202 \\
\hline No & $203(71.5)$ & $162(73.3)$ & $41(65.1)$ & \\
\hline Yes & $81(28.5)$ & $59(26.7)$ & $22(34.9)$ & \\
\hline Reason for extension of stay & & & & 0.001 \\
\hline No extension & $203(72.2)$ & $162(74.0)$ & $41(66.1)$ & \\
\hline Medical & $32(11.4)$ & $20(9.1)$ & $12(19.4)$ & \\
\hline Rehabilitation & $6(2.1)$ & $6(2.7)$ & $0(0.0)$ & \\
\hline Social & $30(10.7)$ & 27 (12.3) & $3(4.8)$ & \\
\hline Social \& Rehabilitation & $1(0.4)$ & $1(0.5)$ & $0(0.0)$ & \\
\hline Others & $9(3.2)$ & $3(1.4)$ & $6(9.7)$ & \\
\hline Feeding tube at discharge & & & & 1.000 \\
\hline No & $279(98.2)$ & $217(98.2)$ & $62(98.4)$ & \\
\hline Yes & $5(1.8)$ & $4(1.8)$ & $1(1.6)$ & \\
\hline Urinary catheter at discharge & & & & 0.246 \\
\hline No & $266(93.7)$ & $209(94.6)$ & $57(90.5)$ & \\
\hline Yes & $18(6.3)$ & $12(5.4)$ & $6(9.5)$ & \\
\hline Diapers at discharge & & & & 0.557 \\
\hline No & $149(52.5)$ & $118(53.4)$ & $31(49.2)$ & \\
\hline Yes & $135(47.5)$ & $103(46.6)$ & $32(50.8)$ & \\
\hline Discharge destination & & & & 0.006 \\
\hline Not home & $38(13.4)$ & $23(10.5)$ & $15(23.8)$ & \\
\hline Home & $245(86.6)$ & $197(89.5)$ & $48(76.2)$ & \\
\hline \multicolumn{5}{|l|}{ Functional assessments } \\
\hline Modified Barthel index on admission & $42 \pm 17.4(0-80)$ & $42.9 \pm 17.8(0-80)$ & $39.3 \pm 16.4(5-68)$ & 0.147 \\
\hline Modified Barthel Index at discharge & $44.7 \pm 23.9(0-97)$ & $44.7 \pm 24.4(0-97)$ & $44.8 \pm 22.6(7-95)$ & 0.971 \\
\hline $\begin{array}{l}\text { Modified Barthel Index efficiency (on discharge } \\
\text { - at admission) / length of stay }\end{array}$ & $-0.3(-0.7-0.0)$ & $-0.3(-0.8-0)$ & $-0.3(-0.7-0)$ & 0.794 \\
\hline Gait speed, m/s & $0.44(0.26-0.57)$ & $0.46(0.31-0.61)$ & $0.36(0.21-0.45)$ & 0.167 \\
\hline Gait speed, m/s & & & & 0.328 \\
\hline$<0.8$ & $65(97.0)$ & $54(98.2)$ & $11(91.7)$ & \\
\hline$\geq 0.8$ & $2(3.0)$ & $1(1.8)$ & $1(8.3)$ & \\
\hline Hand grip strength on admission, $\mathrm{kg}$ & $10.0(7-14.3)$ & $10(7-14.7)$ & $10.6(7.5-14.1)$ & 0.779 \\
\hline Hand grip strength at discharge, kg & $10.5(7.4-14.7)$ & $10.4(7.4-14.6)$ & $11.4(7.6-15.9)$ & 0.491 \\
\hline Change in handgrip strength, $\mathrm{kg}$ & $0.2(-0.2-0.7)$ & $0.2(-0.2-0.7)$ & $0.1(-0.2-0.6)$ & 0.601 \\
\hline Hand grip strength at discharge in men, $\mathrm{kg}$ & $14.5(10.0-18.7)$ & $14.5(10.1-18.5)$ & $14.7(8.4-23.4)$ & 0.633 \\
\hline Hand grip strength at discharged in women, $\mathrm{kg}$ & $9.0(6.8-12.4)$ & $9.0(6.6-12.5)$ & $9.6(7.1-12.7)$ & 0.747 \\
\hline
\end{tabular}


TABLE 2

Factors associated with 30 -days post-discharge unplanned readmission

\begin{tabular}{|c|c|c|c|c|}
\hline \multirow[t]{2}{*}{ Factor } & \multicolumn{2}{|c|}{ Univariate analysis } & \multicolumn{2}{|c|}{ Multivariate logistic regression } \\
\hline & $\begin{array}{l}\text { Odds ratio (95\% } \\
\text { confidence interval) }\end{array}$ & $p$ Value & $\begin{array}{l}\text { Adjusted odds ratio } \\
\text { (95\% confidence } \\
\text { interval) }\end{array}$ & $\mathrm{p}$ Value \\
\hline \multicolumn{5}{|l|}{ Previous myocardial infarction } \\
\hline No & Ref & & Ref & \\
\hline Yes & $1.91(1.05-3.48)$ & 0.035 & $1.73(0.90-3.36)$ & 0.102 \\
\hline \multicolumn{5}{|c|}{ Moderate or severe liver or renal disease } \\
\hline No & Ref & & Ref & \\
\hline Yes & $3.86(1.39-10.73)$ & 0.010 & $1.34(0.58-3.10)$ & 0.491 \\
\hline Charlson Comorbidities Index & $1.20(1.08-1.34)$ & 0.001 & & \\
\hline \multicolumn{5}{|l|}{ Albumin level } \\
\hline Normal & Ref & & Ref & \\
\hline Low & $2.02(1.13-3.59)$ & 0.018 & $1.54(0.83-2.88)$ & 0.171 \\
\hline LACE Index & $1.18(1.05-1.32)$ & 0.004 & $1.05(0.91-1.22)$ & 0.505 \\
\hline \multicolumn{5}{|c|}{ Emergency department visits in prior 6 months } \\
\hline No & Ref & & Ref & \\
\hline Yes & $1.77(1.00-3.12)$ & 0.049 & $0.76(0.30-1.96)$ & 0.575 \\
\hline \multicolumn{5}{|l|}{ Hospitalisation in prior 6 months } \\
\hline No & Ref & & Ref & \\
\hline Yes & $2.42(1.37-4.30)$ & 0.002 & $2.62(1.02-6.75)$ & 0.045 \\
\hline \multicolumn{5}{|l|}{ Discharge destination } \\
\hline Home & Ref & & Ref & \\
\hline Not home & $2.68(1.30-5.52)$ & 0.008 & $3.10(1.38-6.94)$ & 0.006 \\
\hline
\end{tabular}

TABLE 3

Performance of four models in predicting 30 -day post-discharge unplanned readmission*

\begin{tabular}{lrrrr}
\hline Performance & Model 1 & Model 2 & Model 3 & Model 4 \\
\hline Hosmer-Lemeshow goodness-of-fit test & $\mathrm{p}=0.324$ & $\mathrm{p}=0.734$ & $\mathrm{p}=0.753$ & $\mathrm{p}=0.974$ \\
Sensitivity, \% & $31 / 62(50.0)$ & $42 / 62(67.7)$ & $28 / 62(45.2)$ & $44 / 62(71.0)$ \\
Specificity, \% & $183 / 216(84.7)$ & $124 / 220(56.4)$ & $188 / 216(87.0)$ & $121 / 219(55.3)$ \\
Positive predictive value, \% & $31 / 64(48.4)$ & $42 / 138(30.4)$ & $28 / 56(50.0)$ & $44 / 142(31.0)$ \\
Negative predictive value, \% & $183 / 214(85.5)$ & $124 / 144(86.1)$ & $188 / 222(84.7)$ & $121 / 139(87.1)$ \\
$\begin{array}{l}\text { Area under the receiver operating } \\
\text { characteristics curve (95\% Cl) }\end{array}$ & $0.70(0.62-0.78)$ & $0.64(0.56-0.72)$ & $0.70(0.62-0.78)$ & $0.69(0.61-0.76)$ \\
Brier score & & & & 0.152 \\
\hline
\end{tabular}

* Model 1 includes variables of previous myocardial infarction, moderate or severe liver or renal disease, albumin status, ever visited to emergency

department, history of hospitalisation, staying place after discharge, and LACE index; model 2 includes variables of history of hospitalisation, and staying place after discharge; model 3 includes model 1 without LACE index; and model 4 (forward stepwise method) includes variables of previous myocardial infarction, moderate or severe liver or renal disease, history of hospitalisation, and staying place after discharge

\section{DISCUSSION}

There have been studies evaluating predictors of readmission from acute hospitals in Singapore. ${ }^{1,2,20}$ Traditional predictive models include the LACE index and the Charlson Comorbidity Index, but the LACE index was reported to have poor discriminating ability in predicting readmission within 30 days. ${ }^{21} \mathrm{In}$ a systematic review of nine studies, risk factors that were associated with hospital readmission included 


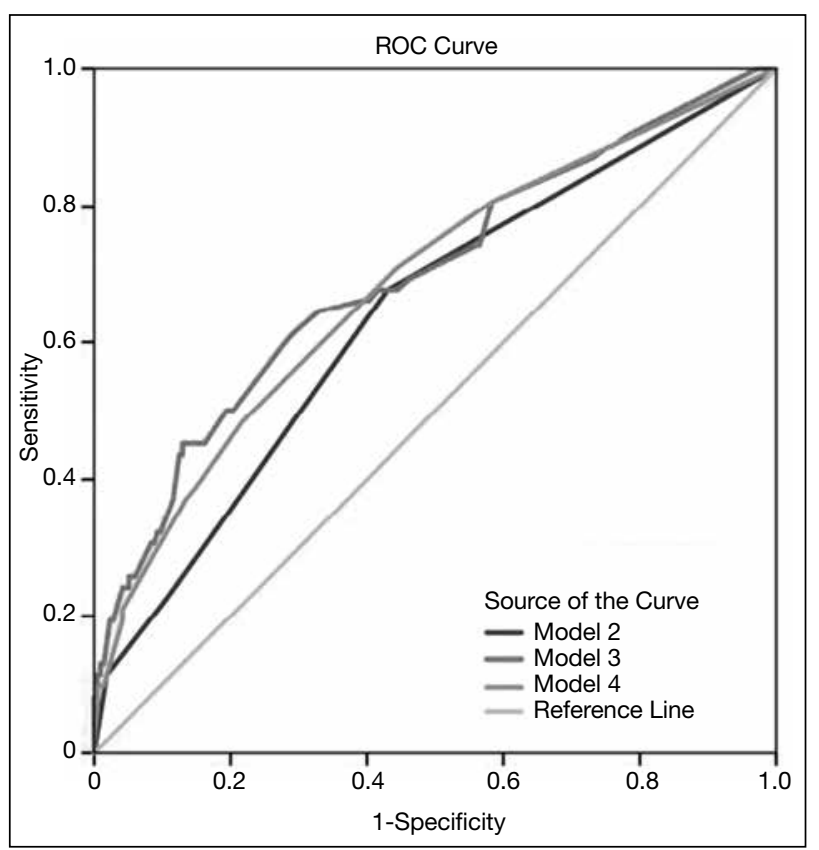

FIGURE. The area under the receiver operating characteristics curve of four models ranges from 0.6 to 0.7 . The discrimination ability of the models was weak.

higher advanced age, male sex, ethnicity, living conditions, impaired health capacity (such as poor overall condition and functional disability), prior admissions, longer length of hospital stays, way of referral, and discharge destination. ${ }^{22}$

In the present study, we included other factors such as functional status, presence of geriatric syndromes, frailty status, and other sociodemographic factors that we postulated to be relevant. ${ }^{23,24}$ Multivariate analysis revealed two independent predictors of unplanned readmission within 30 days: hospitalisation in the prior 6 months and discharge destination other than home.

Early discharge care planning and post-discharge support could benefit this high-risk group who already have poorer health and reserve. Early discharge care planning involves a multidisciplinary team, regular family engagement, and community and social services support. In Singapore, there are hospital avoidance programmes where geriatricians and a multidisciplinary team provide support to patients discharged to selected nursing homes. ${ }^{25}$ These programmes focus on advance care planning and discussions of preferred plan of care to address end-of-life issues. It may be beneficial to broaden the scope and scale of such programmes to cover all nursing homes in the future. Other programmes include the Hospital to Home Service where at-risk patients are referred for continuing social, nursing, functional, and clinical support post-discharge through a multidisciplinary team.

In the present study, functional and frailty statuses were not independent predictors of 30-day post-discharge unplanned readmission. This may be because our patients were severely dependent (mean modified Barthel Index of 44.7 on discharge) and frail (prevalence of frailty of $62.1 \%$ ). Hand grip strength of patients was significantly lower than the normative values of older Singaporeans in a crosssectional study. ${ }^{26}$ The mean hand grip strength was $10.8 \mathrm{~kg}$ to $21.7 \mathrm{~kg}$ for men and $7.8 \mathrm{~kg}$ to $15.3 \mathrm{~kg}$ for women, which are significantly lower than that in the general elderly population.

There are some limitations to our study. The patient population was specific to the subacute geriatric ward of a community hospital, and hence the findings may not be generalisable to all older adults. The sample size was small and may affect the statistical significance of some predictors.

\section{CONCLUSION}

30-day post-discharge unplanned readmission was associated with the LACE index, history of myocardial infarction, moderate or severe renal or liver disease, low serum albumin levels, prior healthcare utilisation (emergency department visits and hospitalisations in the 6 months prior to discharge), and discharge destination. Out of these, hospitalisation in the prior 6 months and discharge destination other than home were independent predictors. However, the discriminative ability of our predictive models was weak. Future studies should include a larger sample size in a multicentre setting.

\section{ACKNOWLEDGEMENTS}

The authors would like to acknowledge the nursing and allied health team of St. Andrew's Community Hospital for their assistance.

\section{FUNDING}

This study is supported by Changi General Hospital, 
Singapore and St. Andrew's Community Hospital, Singapore.

\section{DECLARATION}

The authors have no conflicts of interest to disclose.

\section{REFERENCES}

1. Low LL, Tay WY, Ng MJ, Tan SY, Liu N, Lee KH. Frequent hospital admissions in Singapore: clinical risk factors and impact of socioeconomic status. Singapore Med J 2018;59:39-43. Crossref

2. Low LL, Liu N, Wang S, Thumboo J, Ong ME, Lee KH. Predicting 30-day readmissions in an Asian population: building a predictive model by incorporating markers of hospitalization severity. PLoS One 2016;11:e0167413. Crossref

3. Zhou H, Della PR, Roberts P, Goh L, Dhaliwal SS. Utility of models to predict 28-day or 30-day unplanned hospital readmissions: an updated systematic review. BMJ Open 2016;6:e011060. Crossref

4. Donzé JD, Williams MV, Robinson EJ, et al. International validity of the HOSPITAL score to predict 30-day potentially avoidable hospital readmissions. JAMA Intern Med 2016;176:496502. Crossref

5. Burke RE, Whitfield EA, Hittle D, et al. Hospital readmission from post-acute care facilities: risk factors, timing, and outcomes. J Am Med Dir Assoc 2016;17:249-55. Crossref

6. Sganga F, Landi F, Volpato S, et al. Predictors of rehospitalization among older adults: results of the CRIME Study. Geriatr Gerontol Int 2017;17:1588-92. Crossref

7. Franchi C, Nobili A, Mari D, et al. Risk factors for hospital readmission of elderly patients. Eur J Intern Med 2013;24:4551. Crossref

8. Lanièce I, Couturier P, Dramé M, et al. Incidence and main factors associated with early unplanned hospital readmission among French medical inpatients aged 75 and over admitted through emergency units. Age Ageing 2008;37:416-22. Crossref

9. Singapore health facts-admissions. https://www.moh.gov.sg/ resources-statistics/singapore-health-facts/admissions-andoutpatient-attendances. Assessed 6 January 2020.

10. Community Hospital Care. Handbook for Healthcare Professionals. 2nd ed. Ministry of Health, Singapore. https:// www.moh.gov.sg/docs/librariesprovider5/default-documentlibrary/handbook-on-ch-care-for-healthcare-professionals2nd-edition-200918-for.pdf. Assessed 27 March 2020.

11. Vellas B, Villars H, Abellan G, et al. Overview of the MNA--its history and challenges. J Nutr Health Aging 2006;10:456-65.

12. Ng TP, Niti M, Chiam PC, Kua EH. Ethnic and educational differences in cognitive test performance on mini-mental state examination in Asians. Am J Geriatr Psychiatry 2007;15:130-
9. Crossref

13. Rockwood K, Song X, MacKnight C, et al. A global clinical measure of fitness and frailty in elderly people. CMAJ 2005;173:489-95. Crossref

14. Morley JE, Malmstrom TK, Miller DK. A simple frailty questionnaire (FRAIL) predicts outcomes in middle aged African Americans. J Nutr Health Aging 2012;16:601-8. Crossref

15. Shah S, Vanclay F, Cooper B. Improving the sensitivity of the Barthel Index for stroke rehabilitation. J Clin Epidemiol 1989;42:703-9. Crossref

16. Roberts HC, Denison HJ, Martin HJ, et al. A review of the measurement of grip strength in clinical and epidemiological studies: towards a standardised approach. Age Ageing 2011;40:423-9. Crossref

17. Braden HJ, Hilgenberg S, Bohannon RW, Ko MS, Hasson S. Gait speed is limited but improves over the course of acute care physical therapy. J Geriatr Phys Ther 2012;35:140-4. Crossref

18. Charlson ME, Pompei P, Ales KL, MacKenzie CR. A new method of classifying prognostic comorbidity in longitudinal studies: development and validation. J Chronic Dis 1987;40:37383. Crossref

19. van Walraven C, Dhalla IA, Bell C, et al. Derivation and validation of an index to predict early death or unplanned readmission after discharge from hospital to the community. CMAJ 2010;182:5517. Crossref

20. Tan RRQ, Toh HJ, Yap PLK, Jiao N, Wang W. 90-day hospital readmissions of patients in a geriatric acute care ward in Singapore. Clin Nurs Res 2020;29:200-9. Crossref

21. Low LL, Liu N, Ong MEH, et al. Performance of the LACE index to identify elderly patients at high risk for hospital readmission in Singapore. Medicine (Baltimore) 2017;96:e6728. Crossref

22. Pedersen MK, Meyer G, Uhrenfeldt L. Risk factors for acute care hospital readmission in older persons in Western countries: a systematic review. JBI Database System Rev Implement Rep 2017;15:454-85. Crossref

23. Lehn SF, Zwisler AD, Pedersen SGH, Gjørup T, Thygesen LC. Development of a prediction model for 30 -day acute readmissions among older medical patients: the influence of social factors along with other patient-specific and organisational factors. BMJ Open Qual 2019;8:e000544. Crossref

24. Iloabuchi TC, Mi D, Tu W, Counsell SR. Risk factors for early hospital readmission in low-income elderly adults. J Am Geriatr Soc 2014;62:489-94. Crossref

25. Link in Centres \& Services Geriatric Medicine. Annual Report 2016-2017. Changi General Hospital. https://www.cgh.com. sg/centres-services/Pages/Geriatric-Medicine.aspx. Assessed 4 March 2020.

26. Malhotra R, Ang S, Allen JC, et al. Normative values of hand grip strength for elderly Singaporeans aged 60 to 89 years: a cross-sectional study. J Am Med Dir Assoc 2016;17:864.e1-864. e8647. Crossref 